\title{
A DECOMPOSITION FORMULA FOR THE WEIGHTED COMMUTATOR
}

\author{
NELSON MARTINS-FERREIRA AND TIM VAN DER LINDEN \\ Dedicated to George Janelidze on the occasion of his sixtieth birthday
}

Abstract. We decompose the weighted subobject commutator of M. Gran, G. Janelidze and A. Ursini as a join of a binary and a ternary commutator.

In their article [6], M. Gran, G. Janelidze and A. Ursini introduce a weighted normal commutator which, depending on the chosen weight, captures classical commutators such as the Huq commutator [9, 3, 1] and the Smith commutator [15, 14, 4, 1,. It is constructed as the normal closure of a so-called weighted subobject commutator. We show how this latter commutator may be decomposed as a join of a binary and a ternary commutator [8, 7] defined in terms of co-smash products [5]. We moreover explain that the corresponding concept of weighted centrality of arrows can be expressed in terms of the admissibility of certain diagrams in the first author's sense [12].

The weighted subobject commutator. In a finitely cocomplete homological category [1, 10], a weighted cospan is a triple of morphisms

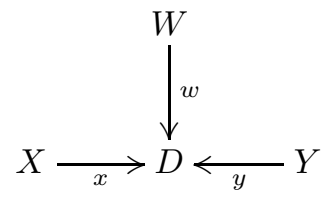

in which $(x, y)$ plays the role of cospan and $w$ is the weight. Consider the pullback

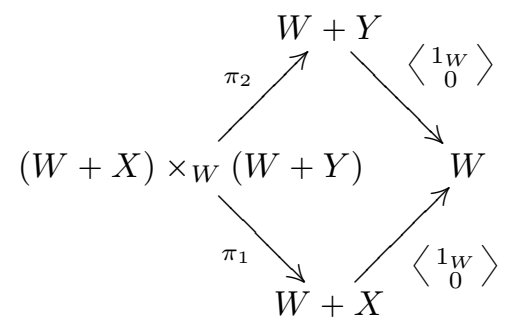

Date: 6th September 2021.

2010 Mathematics Subject Classification. 18E10, 08A30.

Key words and phrases. Semi-abelian, finitely cocomplete homological category; weighted, higher-order commutator; co-smash product.

The first author was supported by IPLeiria/ESTG-CDRSP and Fundação para a Ciência e a Tecnologia (grants SFRH/BPD/4321/2008 and PTDC/MAT/120222/2010).

The second author works as chargé de recherches for Fonds de la Recherche ScientifiqueFNRS. His research was supported by Centro de Matemática da Universidade de Coimbra and by Fundação para a Ciência e a Tecnologia (grant SFRH/BPD/38797/2007). He wishes to thank CMUC, IPLeiria and UCT for their kind hospitality during his stays in Coimbra, in Leiria and in Cape Town. 
and the induced outer diagram

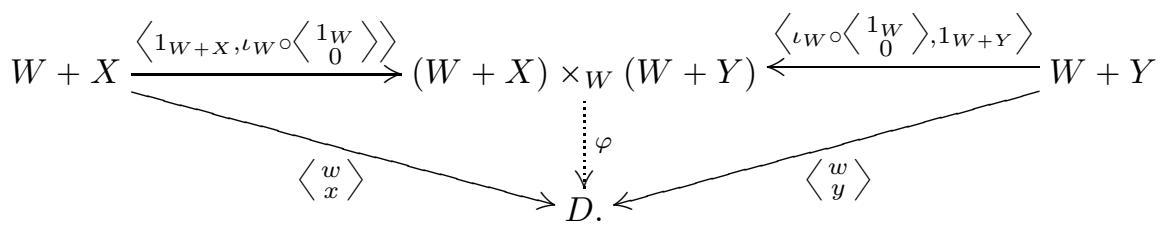

In [6] the morphisms $x$ and $y$ are said to commute over $w$ if and only if there exists a dotted arrow $\varphi$ (called an internal multiplication) such that the above diagram is commutative.

As explained in [6], taking $W=0$ captures commuting pairs in the Huq sense ( $x$ and $y$ commute over 0 if and only if they Huq-commute), and $w=1_{D}$ captures centralising equivalence relations in the Smith sense (the respective normalisations $x$ and $y$ of two equivalence relations $R$ and $S$ on $D$ commute over $1_{D}$ if and only if $R$ and $S$ Smith-commute).

Now consider the canonical comparison morphism

$$
\left\langle\begin{array}{cc}
\iota_{W} & \iota_{W} \\
\iota_{X} & 0 \\
0 & \iota_{Y}
\end{array}\right\rangle: W+X+Y \rightarrow(W+X) \times_{W}(W+Y)
$$

which, being a regular epimorphism [6] as the comparison between a sum and a product in the category of points over an object $W$ in a regular Mal'tsev category, induces a short exact sequence

$$
0 \longrightarrow K \longmapsto W+X+Y \stackrel{\left\langle\begin{array}{ccc}
\iota_{W} & \iota_{W} \\
\iota_{X} & 0 \\
0 & \iota_{Y}
\end{array}\right.}{\longrightarrow}(W+X) \times_{W}(W+Y) \longrightarrow 0
$$

The $(W, w)$-weighted subobject commutator $\kappa:[(X, x),(Y, y)]_{(W, w)} \rightarrow D$ of $x$ and $y$ is the direct image of $K$ along the induced arrow to $D$ as in

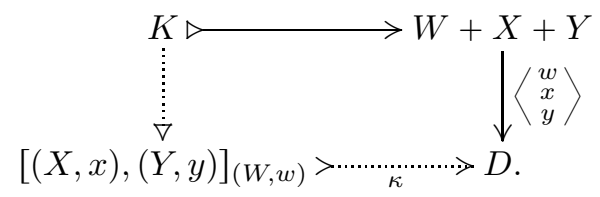

It is clear from the exactness of the above sequence that $x$ and $y$ commute over $w$ if and only if $[(X, x),(Y, y)]_{(W, w)}$ vanishes.

The normal closure of $\kappa$ is called the $(W, w)$-weighted normal commutator of $x$ and $y$ and denoted by $N[(X, x),(Y, y)]_{(W, w)}$.

Admissibility. In order to analyse the weighted subobject commutator in terms of the binary and ternary commutators considered in [8, 7, we pass via an intermediate notion from [12. An admissibility diagram is a diagram of shape

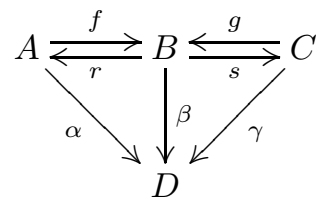


with $f \circ r=1_{B}=g \circ s$ and $\alpha \circ r=\beta=\gamma \circ s$. Note that by taking the pullback of $f$ with $g$, any admissibility diagram such as (C) may be extended to

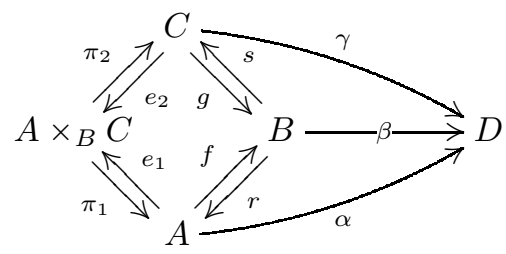

in which the pullback square is a double split epimorphism.

The triple $(\alpha, \beta, \gamma)$ is said to be admissible with respect to $(f, r, g, s)$ if there is a (necessarily unique) morphism $\varphi: A \times{ }_{B} C \rightarrow D$ such that $\varphi \circ e_{1}=\alpha$ and $\varphi \circ e_{2}=\gamma$.

Commuting pairs in terms of admissibility. It is immediately clear from the definitions that the morphisms $x$ and $y$ commute over $w$ if and only if the triple $\left(\left\langle\begin{array}{c}w \\ x\end{array}\right\rangle, w,\left\langle\begin{array}{c}w \\ y\end{array}\right\rangle\right)$ is admissible with respect to $\left(\left\langle\begin{array}{c}1_{W} \\ 0\end{array}\right\rangle, \iota_{W},\left\langle\begin{array}{c}1_{W} \\ 0\end{array}\right\rangle, \iota_{W}\right)$ as in the diagram

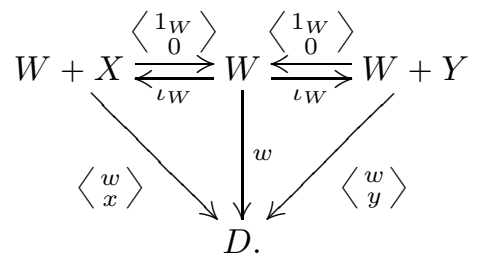

Admissibility in terms of commuting pairs. Consider a diagram (C) and the induced weighted cospan

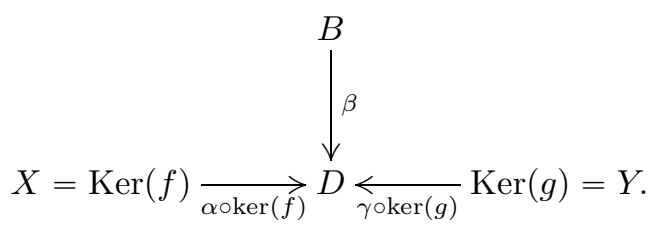

We claim that the triple $(\alpha, \beta, \gamma)$ is admissible with respect to $(f, r, g, s)$ if and only if $x=\alpha \circ \operatorname{ker}(f)$ and $y=\gamma \operatorname{ker}(g)$ commute over $w=\beta: W=B \rightarrow D$. To see this, it suffices to compare Diagram (C) with the induced Diagram $(\underline{D})$. In fact there is a regular epimorphism of admissibility diagrams from the latter to the former which keeps $D$ fixed and makes

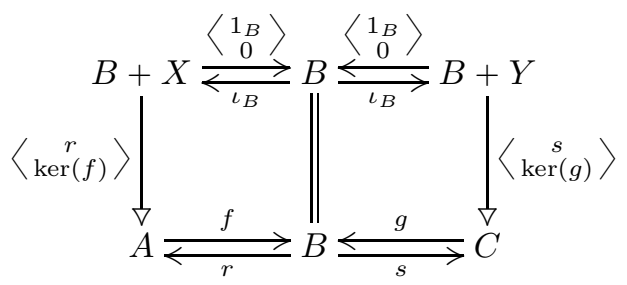

commute. This already proves the "only if" in our claim. For the "if" suppose that $x$ and $y$ commute over $\beta$. For the induced arrow

$$
\varphi:(B+X) \times_{B}(B+Y) \rightarrow D
$$

to factor over the regular epimorphism

$$
\left\langle\begin{array}{r}
\stackrel{r}{\operatorname{ker}(f)}
\end{array}\right\rangle \times{ }_{B}\left\langle\begin{array}{c}
s \\
\operatorname{ker}(g)
\end{array}\right\rangle:(B+X) \times{ }_{B}(B+Y) \rightarrow A \times_{B} C,
$$


we only need that it vanishes on $\operatorname{Ker}(\langle\underset{\operatorname{ker}(f)}{r}\rangle) \times \operatorname{Ker}(\langle\underset{\operatorname{ker}(g)}{s}\rangle)$. This does indeed happen, as

$$
\begin{aligned}
\varphi \circ(\operatorname{ker}(\langle\underset{\operatorname{ker}(f)}{r})) \times \operatorname{ker}(\langle\underset{s}{s}(g)\rangle)) \circ\langle 1,0\rangle & =\varphi \circ\left\langle 1_{B+X}, \iota_{B} \circ\left\langle\begin{array}{c}
1_{B} \\
0
\end{array}\right\rangle\right\rangle \circ \operatorname{ker}(\langle\underset{\operatorname{ker}(f)}{r}\rangle) \\
& =\left\langle\begin{array}{c}
\beta \\
x
\end{array}\right\rangle \circ \operatorname{ker}(\langle\underset{\operatorname{ker}(f)}{r}\rangle) \\
& =\alpha \circ\langle\underset{\operatorname{ker}(f)}{r}\rangle \operatorname{ker}(\langle\underset{\operatorname{ker}(f)}{r}\rangle)
\end{aligned}
$$

is trivial. Similarly, one can check that the arrow

$$
\varphi \circ(\operatorname{ker}(\langle\underset{\operatorname{ker}(f)}{\stackrel{r}{ }}\rangle) \times \operatorname{ker}(\langle\underset{\operatorname{ker}(g)}{s}\rangle)) \circ\langle 0,1\rangle
$$

is trivial.

Binary and ternary Higgins commutators. If $k: K \rightarrow X$ and $l: L \rightarrow X$ are subobjects of an object $X$ in a finitely cocomplete homological category, then the (Higgins) commutator $[K, L] \leqslant X$ is the image of the induced morphism

$$
K \diamond L \stackrel{\iota_{K, L}}{\longrightarrow} K+L \stackrel{\left\langle\begin{array}{l}
k \\
l
\end{array}\right\rangle}{\longrightarrow} X,
$$

where

$$
K \diamond L=\operatorname{Ker}\left(\left\langle\begin{array}{cc}
1_{K} & 0 \\
0 & 1_{L}
\end{array}\right\rangle: K+L \rightarrow K \times L\right) .
$$

As explained in [6], the Higgins commutator is another special case of the weighted subobject commutator recalled above. This commutator was first introduced in [7, 11. Higher-order versions of it exist and are studied in [8, 7].

The object $K \diamond L$, as the $K \diamond L \diamond M$ below, is an example of a co-smash product $[5]$. It is worth recalling form [11 that it may be computed as the intersection $K b L \wedge L b K$, where the object $K b L$ from [2] is the kernel in the split exact sequence

$$
0 \longrightarrow K b L \longmapsto K+L \stackrel{\left\langle\begin{array}{c}
1_{K} \\
0
\end{array}\right\rangle}{\underset{\iota_{K}}{\longleftrightarrow}} K \longrightarrow 0 .
$$

Furthermore, also the sequence

$$
0 \longrightarrow K \diamond L \longmapsto K b L \rightleftarrows L
$$

is split exact.

If $m: M \rightarrow X$ is another subobject of $X$, then the ternary commutator $[K, L, M] \leqslant X$ is defined as the image of the composite

$$
K \diamond L \diamond M \stackrel{\iota_{K, L, M}}{\longrightarrow} K+L+M \stackrel{\left\langle\begin{array}{c}
k \\
l \\
m
\end{array}\right\rangle}{\longrightarrow} X,
$$

where $\iota_{K, L, M}$ is the kernel of the morphism

$$
K+L+M \stackrel{\left\langle\begin{array}{ccc}
i_{K} & i_{K} & 0 \\
i_{L} & 0 & i_{L} \\
0 & i_{M} & i_{M}
\end{array}\right\rangle}{\longrightarrow}(K+L) \times(K+M) \times(L+M) .
$$

It is well known that co-smash products are not associative, in general; furthermore, ternary co-smash products or commutators need not be decomposable into iterated binary ones: see [5, 7, 8].

Theorem 1. Consider a weighted cospan (A) such that $x$ and $y$ are normal monomorphisms (= kernels) in a finitely cocomplete homological category. Then $x$ and $y$ commute over $w$ precisely when the commutators $[X, Y]$ and $[X, Y, \operatorname{Im}(w)]$ vanish. 
Proof. First of all we show that $x$ and $y$ coincide with the images of $\left\langle\begin{array}{c}w \\ x\end{array}\right\rangle$ oker $\left(\left\langle\begin{array}{c}1_{W} \\ 0\end{array}\right\rangle\right)$ and $\left\langle\begin{array}{l}w \\ y\end{array}\right\rangle \operatorname{ker}\left(\left\langle\begin{array}{c}1_{W} \\ 0\end{array}\right\rangle\right)$, respectively, as in (D). To see this, we consider the diagram with short exact rows

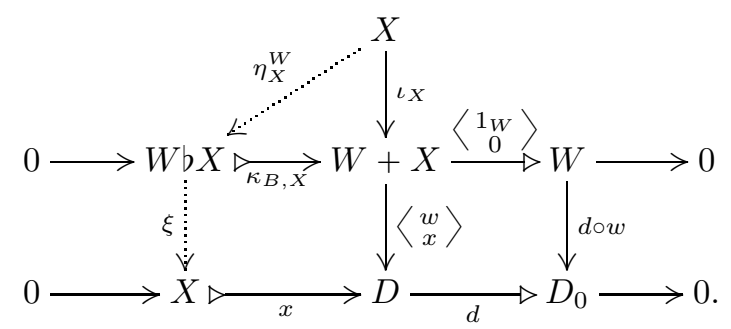

It is clear that $\left\langle\begin{array}{c}1_{W} \\ 0\end{array}\right\rangle \circ \iota_{X}=0$ induces the factorisation $\eta_{X}^{W}$ of $\iota_{X}$ over the kernel $\kappa_{B, X}$ of $\left\langle\begin{array}{c}1_{W} \\ 0\end{array}\right\rangle$. Similarly, since

$$
d \circ\left\langle\begin{array}{l}
w \\
x
\end{array}\right\rangle \circ \kappa_{B, X}=d \circ w \circ\left\langle\begin{array}{c}
1_{W} \\
0
\end{array}\right\rangle \circ \kappa_{B, X}
$$

is trivial we obtain the dotted factorisation $\xi$. Now

$$
x \circ \xi \circ \eta_{X}^{W}=\left\langle\begin{array}{l}
w \\
x
\end{array}\right\rangle \circ \kappa_{B, X} \circ \eta_{X}^{W}=\left\langle\begin{array}{l}
w \\
x
\end{array}\right\rangle \circ \iota_{X}=x,
$$

so $\xi \circ \eta_{X}^{W}=1_{X}$ because $x$ is a monomorphism. In particular, $\xi$ is a regular epimorphism. It follows that $x$ is the image of $\left\langle\begin{array}{c}w \\ x\end{array}\right\rangle \circ \kappa_{B, X}$.

We know from the above discussion that $x$ and $y$ commute over $w$ precisely when the triple $\left(\left\langle\begin{array}{c}w \\ x\end{array}\right\rangle, w,\left\langle\begin{array}{l}w \\ y\end{array}\right\rangle\right)$ is admissible with respect to $\left(\left\langle\begin{array}{c}1_{W} \\ 0\end{array}\right\rangle, \iota_{W},\left\langle\begin{array}{c}1_{W} \\ 0\end{array}\right\rangle, \iota_{W}\right)$. Lemma 4.5 in 8 now tells us that this happens if and only if the commutators $[X, Y]$ and $[X, Y, \operatorname{Im}(w)]$ vanish.

Via Theorem 4.6 in [8] we now recover the known result that the Smith is Huq condition [13] holds if and only if, for any given cospan of normal monomorphisms $(x, y)$, the property of commuting over $w$ is independent of the chosen weight $w$ making $(x, y, w)$ a weighted cospan.

We also see that the $(W, w)$-weighted normal commutator $N[(X, x),(Y, y)]_{(W, w)}$ of $x$ and $y$ is the normal closure of $[X, Y] \vee[X, Y, \operatorname{Im}(w)]$ in $D$, since these two normal subobjects satisfy the same universal property. We shall, however, not insist further on this, because we can obtain the following refinement (Theorem 2).

Lemma 1. If $X, Y$, and $W$ are objects in a finitely cocomplete homological category, then there is a decomposition

$$
(X+Y) \diamond W \cong((X \diamond Y \diamond W) \rtimes(X \diamond W)) \rtimes(Y \diamond W) .
$$

More precisely, there exists an object $V$ and split short exact sequences

$$
0 \longrightarrow V \longmapsto(X+Y) \diamond W \longleftrightarrow Y \diamond W \longrightarrow 0
$$

and

$$
0 \longrightarrow X \diamond Y \diamond W \triangleright X \diamond W \longrightarrow 0
$$

Proof. This is Lemma 2.12 in [8], a result which was first obtained by M. Hartl and B. Loiseau.

Theorem 2. Given a weighted cospan (A) in a finitely cocomplete homological category, the $(W, w)$-weighted subobject commutator of $x$ and $y$ decomposes as

$$
[(X, x),(Y, y)]_{(W, w)}=[X, Y] \vee[X, Y, \operatorname{Im}(w)] .
$$


Proof. We decompose the kernel $K$ of the short exact sequence (B) into a join of the co-smash products $X \diamond Y$ and $X \diamond Y \diamond W$ considered as subobjects of $K$. The result then follows from the compatibility of the ternary co-smash product with image factorisations (Corollary 2.14 in [7] and the fact that co-smash products preserve monomorphisms). Indeed, the image of the composite $X \diamond Y \diamond W \rightarrow W+X+Y \rightarrow D$ is $[X, Y, \operatorname{Im}(w)]$, which is a subobject of $[(X, x),(Y, y)]_{(W, w)}$. It is easily seen that also $[X, Y] \leqslant[(X, x),(Y, y)]_{(W, w)}$ and that these two inclusions are jointly regular epic.

Consider the cube of solid split epimorphisms

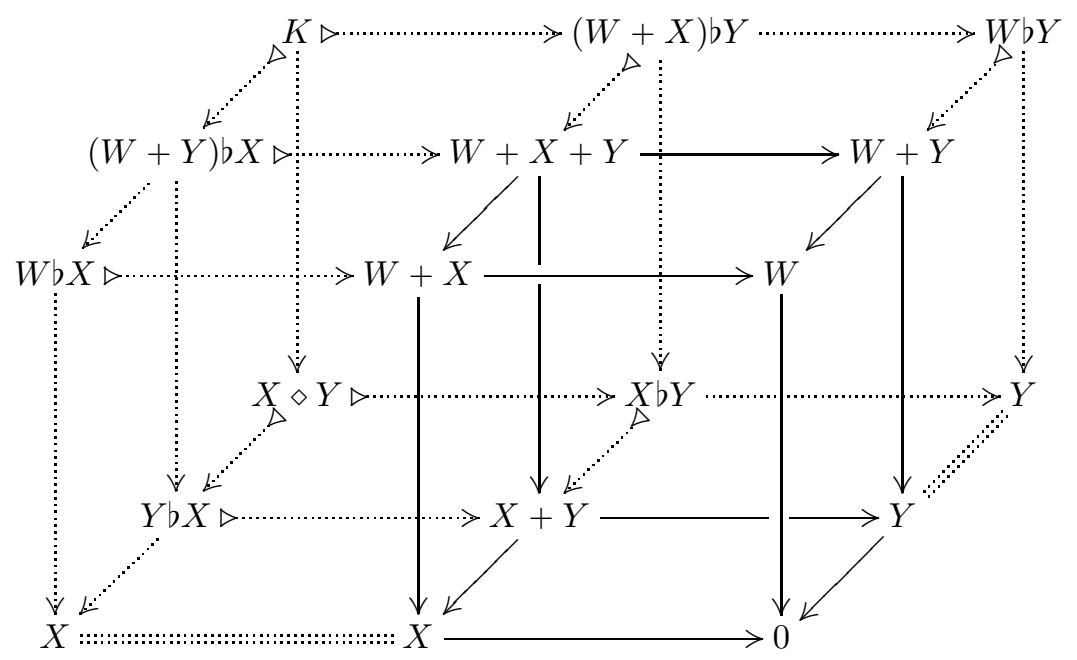

which, taking kernels horizontally, yields two $3 \times 3$ diagrams (or, equivalently, a $3 \times 3$ diagram of vertical split epimorphisms). Note that the bottom one has $X \diamond Y$, and the top one $K$, in its back left corner. It suffices to prove that, taking kernels vertically now, we obtain the split exact sequence

$$
0 \longrightarrow X \diamond Y \diamond W \longmapsto X \longmapsto Y \gg X
$$

in the back left corner of the induced $3 \times 3 \times 3$ diagram. Taking vertical kernels of the front and middle sections of the diagram above, we already obtain a morphism

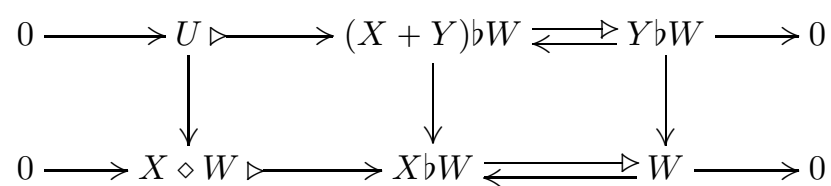

of short exact sequences. Using $(\mathbf{E})$ we see that the sequence

$$
0 \longrightarrow U \bowtie(X+Y) \diamond W \longleftrightarrow Y \diamond W \longrightarrow 0
$$

is split exact. Noting that $V$ in Lemma 1 is the object $U$, we see that the co-smash product $X \diamond Y \diamond W$ must coincide with the kernel of $U \rightarrow X \diamond W$, which we already know coincides with the needed kernel of $K \rightarrow X \diamond Y$.

Acknowledgement. We would like to thank the referee who guided us through a major revision of the article and thus improved the presentation considerably.

\section{REFERENCES}

[1] F. Borceux and D. Bourn, Mal'cev, protomodular, homological and semi-abelian categories, Math. Appl., vol. 566, Kluwer Acad. Publ., 2004.

[2] F. Borceux, G. Janelidze, and G. M. Kelly, Internal object actions, Comment. Math. Univ. Carolinae 46 (2005), no. 2, 235-255. 
[3] D. Bourn, Commutator theory in regular Mal'cev categories, Galois Theory, Hopf Algebras, and Semiabelian Categories (G. Janelidze, B. Pareigis, and W. Tholen, eds.), Fields Inst. Commun., vol. 43, Amer. Math. Soc., 2004, pp. 61-76.

[4] D. Bourn and M. Gran, Centrality and connectors in Maltsev categories, Algebra Universalis 48 (2002), 309-331.

[5] A. Carboni and G. Janelidze, Smash product of pointed objects in lextensive categories, J. Pure Appl. Algebra 183 (2003), 27-43.

[6] M. Gran, G. Janelidze, and A. Ursini, Weighted commutators in semi-abelian categories, preprint Séminaire de Mathématique No. 379, Université catholique de Louvain, 2012.

[7] M. Hartl and B. Loiseau, On actions and strict actions in homological categories, Theory Appl. Categ. 27 (2013), no. 15, 347-392.

[8] M. Hartl and T. Van der Linden, The ternary commutator obstruction for internal crossed modules, Adv. Math. 232 (2013), no. 1, 571-607.

[9] S. A. Huq, Commutator, nilpotency and solvability in categories, Quart. J. Math. Oxford 19 (1968), no. 2, 363-389

[10] G. Janelidze, L. Márki, and W. Tholen, Semi-abelian categories, J. Pure Appl. Algebra 168 (2002), no. 2-3, 367-386.

[11] S. Mantovani and G. Metere, Normalities and commutators, J. Algebra 324 (2010), no. 9, $2568-2588$.

[12] N. Martins-Ferreira, Low-dimensional internal categorical structures in weakly Mal'cev sesquicategories, Ph.D. thesis, University of Cape Town, 2008.

[13] N. Martins-Ferreira and T. Van der Linden, A note on the "Smith is Huq" condition, Appl. Categ. Structures 20 (2012), no. 2, 175-187.

[14] M. C. Pedicchio, A categorical approach to commutator theory, J. Algebra 177 (1995), 647657.

[15] J. D. H. Smith, Mal'cev varieties, Lecture Notes in Math., vol. 554, Springer, 1976.

E-mail address: martins.ferreira@ipleiria.pt

E-mail address: tim.vanderlinden@uclouvain.be

Departamento de Matemática, Escola Superior de Tecnologia e Gestão, Centro para o Desenvolvimento Rápido e Sustentado do Produto, Instituto Politécnico de Leiria, Leiria, Portugal

CMUC, Department of Mathematics, University of Coimbra, 3001-454 Coimbra, Portugal

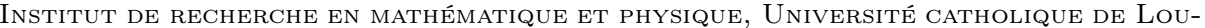
vain, Chemin Du CyClotron 2 Bte L7.01.02, B-1348 Louvain-LA-Neuve, Belgium 